\title{
Automatic Structure Detection in Constraints of Tabular Data
}

\author{
Jordi Castro $^{1, \star, \star \star}$ and Daniel Baena ${ }^{2}$ \\ ${ }^{1}$ Department of Statistics and Operations Research, \\ Universitat Politècnica de Catalunya, \\ Jordi Girona 1-3, 08034 Barcelona, Catalonia \\ jordi.castro@upc.edu \\ http://www-eio.upc.es/ jcastro \\ ${ }^{2}$ Institut d'Estadística de Catalunya, \\ Via Laietana 58, 08003 Barcelona, Catalonia \\ dbaena@idescat.net
}

\begin{abstract}
Methods for the protection of statistical tabular data-as controlled tabular adjustment, cell suppression, or controlled roundingneed to solve several linear programming subproblems. For large multidimensional linked and hierarchical tables, such subproblems turn out to be computationally challenging. One of the techniques used to reduce the solution time of mathematical programming problems is to exploit the constraints structure using some specialized algorithm. Two of the most usual structures are block-angular matrices with either linking rows (primal block-angular structure) or linking columns (dual block-angular structure). Although constraints associated to tabular data have intrinsically a lot of structure, current software for tabular data protection neither detail nor exploit it, and simply provide a single matrix, or at most a set of smallest submatrices. We provide in this work an efficient tool for the automatic detection of primal or dual block-angular structure in constraints matrices. We test it on some of the complex CSPLIB instances, showing that when the number of linking rows or columns is small, the computational savings are significant.
\end{abstract}

Keywords: statistical disclosure control, cell suppression, controlled tabular adjustment, linear constraints, multilevel matrix ordering algorithms.

\section{Introduction}

From an algorithmic point of view, one of the main differences between disclosure control techniques for microdata and tabular data is that the latter must deal with many linear constraints, associated to total and subtotal cells. Current methods for tabular data protection, such as, e.g., cell suppression [4914, controlled tabular adjustment [6]12] and controlled rounding [10 20], deal with

\footnotetext{
* Supported by the Spanish MCyT Project TIC2003-00997.

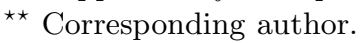

J. Domingo-Ferrer and L. Franconi (Eds.): PSD 2006, LNCS 4302, pp. 12 2006.

(C) Springer-Verlag Berlin Heidelberg 2006
} 
those linear additivity constraints through mathematical programming technology. Unfortunately, the resulting optimization problems turn out to be computationally expensive, even for continuous variables. For instance, the simplex algorithm, which is the preferred option for many linear programming problems 2], has shown to be inefficient compared to polynomial-time interior-point algorithms 21] when dealing with tabular data constraints 6[20.

One of the most used techniques in mathematical programming for reducing the computational cost of a problem is to exploit its structure, either through decomposition or partitioned basis factorization. Two of the most relevant structures are primal block-angular

$$
A=\left[\begin{array}{lllll}
A_{1} & & & \\
& A_{2} & & \\
& & \ddots & \\
& & & A_{k} \\
& L_{1} & L_{2} & \ldots & L_{k}
\end{array}\right],
$$

where $k$ is the number of diagonal blocks, $A \in \mathbb{R}^{m \times n}, A_{i} \in \mathbb{R}^{m_{i} \times n_{i}}, L_{i} \in$ $\mathbb{R}^{l \times n_{i}}, i=1, \ldots, k$, and dual block-angular

$$
A=\left[\begin{array}{ccccc}
A_{1} & & & L_{1} \\
& A_{2} & & L_{2} \\
& & \ddots & \vdots \\
& & & A_{k} & L_{k}
\end{array}\right],
$$

$k, A, A_{i}$ being as before, and $L_{i} \in \mathbb{R}^{m_{i} \times l}$. Structures (11) and (2) appear in problems with $l$ linking constraints and $l$ linking variables, respectively, and have been extensively studied in the literature [3, Chapter 12]. Classical decomposition procedures, based on the simplex method, are Dantzig-Wolfe for primal blockangular structures [13, and Benders for dual block-angular ones [1. Specialized interior-point approaches for structured problems have also been recently suggested [815; these are promising approaches for tabular data protection, since, as noted above, interior-point methods outperform simplex implementations in this class of problems. It is worth noting that homogeneous sizes for diagonal blocks $A_{i}$ benefit the performance of any decomposition approach, mainly if some sort of parallelism is going to be applied.

Unfortunately, current methods and software for tabular data protection do not exploit constraints structure in general tables. Structure has only been exploited for two-dimensional tables with at most one hierarchical variable, whose constraints are modeled as a network [79], and for three-dimensional tables, that provide multicommodity network models [5]. For general tables, state-ofthe-art protection software, as $\tau$-Argus [17, provide a single constraints matrix, or at most a set of linked submatrices, without detailing each matrix structure. Indeed, the constraints structure is particular to each kind of table, and it is not clear that fully exploiting such structure would be worthy for an optimization algorithm. In addition, writing software for the detection of the particular 
structure of any table would be a cumbersome task. The purpose of this work is to overcome such difficulties, i.e., to develop and test a tool for automatic detection of structures (1) and (2) in constraints matrices derived from tabular data. The tool developed is based on the multilevel matrix ordering algorithm for unsymmetric matrices of [16]. Unlike other highly recognized and efficient algorithms, such as that implemented in METIS [18, the former is tailored for unsymmetric matrices, which is the case for tabular data constraints. The procedure is applied to a set of standard tabular data instances, being its behaviour instance dependent: for most instances and $k=2$ a small linking block (i.e., $l / m(l / n)$ for primal (dual) block-angular structures is less than 0.2 ), whereas for others the relative size of the linking block can be up to 0.4 (for $k>2$ these relative sizes of the linking block grow). When the relative size of the linking block is small it makes sense to apply a decomposition approach, and we provide preliminary computational results comparing the performance of a linear programming solver depending on whether structure is exploited.

The paper is organized as follows. Section 2 outlines the multilevel matrix reordering algorithm and gives details about its implementation. Section 3 reports results using this algorithm on a standard set of tabular data instances. Finally, Section 4 analyzes the computational savings due to using the reordered matrix in a mathematical programming solver.

\section{The Matrix Reordering Algorithm}

Given any matrix, obtaining the optimal reordering that transforms the matrix into either the primal block-angular structure (1) or the dual one (2) is a difficult combinatorial optimization problem (in this context, "optimal" means "with the smallest linking block"). Several heuristics have been provided in the past for this problem. We have chosen the recent multilevel approach of [16], which is among the most efficient ones for unsymmetric matrices, and it is implemented in commercial libraries (e.g., in routine HSL_MC66L of the HSL archive, formerly the Harwell Subroutine Library). A comprehensive description of such algorithm is out of the scope of this work, and it will just be outlined; details can be found in [16] and references therein.

We first define some concepts to be used later in the overview of the algorithm. Given a sparse matrix $A \in \mathbb{R}^{m \times n}$, the net of column $j$ is the number of rows associated to such column, i.e, $\left\{i \in\{1, \ldots, m\}: a_{i j} \neq 0\right\}$. A row partition is a partition of the set of rows $\{1, \ldots, n\}$, i.e., $R_{1}, R_{2}$ such that $R_{1} \cap R_{2}=\emptyset$ and $R_{1} \cup R_{2}=\{1, \ldots, m\}$. A net is cut by a row partition if there are rows of the net in both $R_{1}$ and $R_{2}$. The net-cut of a row partition is the number of nets cut by this row partition. Note that in a block-diagonal matrix, i.e, without neither linking constraints nor linking columns, the net-cut is zero. Therefore this is the value to be reduced by any heuristic in order to obtain close-to-block-angular structures. The gain is the decrement of the net-cut obtained after moving a row from $R_{1}$ to $R_{2}$ or vice-versa; the gain is negative if the net-cut is increased. 
The edge-weight of rows $\left(i_{1}, i_{2}\right)$ is the number of columns shared by these rows, i.e, the cardinality of $\left\{j \in\{1, \ldots, n\}: a_{i_{1}, j} \neq 0\right.$ and $\left.a_{i_{2}, j} \neq 0\right\}$.

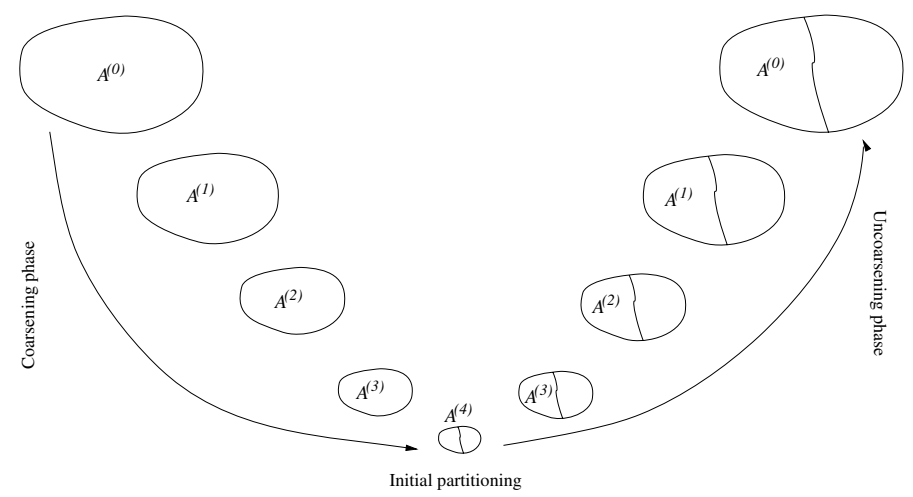

Fig. 1. The three stages of the multilevel ordering algorithm: coarsening, partitioning, uncoarsening; example with 4 levels

The multilevel ordering algorithm of [16] is made of the three following stages, which are shown in Figure 1 .

Coarsening phase. Matrix $A=A^{(0)}$ is successively transformed in a sequence of smaller matrices $A^{(1)}, A^{(2)}, \ldots, A^{(r)}, r$ being the deepest level, such that the number of rows is reduced at each transformation, i.e., $m=m^{(0)}>$ $m^{(1)}>m^{(2)}>\ldots>m^{(r)}$. The procedure successively collapses the "closest rows" in a single one using the notion of edge-weight defined above. In our particular implementation we used the heavy-edge matching criterion of [18 (see that reference for details).

Partitioning phase. It is based on the classical Kernighan-Lin (KL) heuristic [19] for partitioning graphs. Graphs are known to be associated to symmetric sparse matrices, i.e., $a_{i j} \neq 0$ and $a_{j i} \neq 0$ if there is an edge joining nodes $i$ and $j$ in the graph. The KL heuristic can be extended for unsymmetric matrices if we use the notion of net-cut and gain, instead of the original one of edge-cut (i.e., number of edges in a graph cut by a node partition) of the KL algorithm. In short, the KL algorithm is an iterative procedure that starting from an initial row partition $R_{1}^{(0)}, R_{2}^{(0)}$, performs two nested loops. The inner iterations successively look for the row with the largest gain. This row is obtained from the set $R_{1}^{(0)}$ or $R_{2}^{(0)}$ with the maximum cardinality, in an attempt to guarantee similar dimensions for diagonal blocks. This row is moved to the other subset of rows, subsets $R_{1}^{(i)}, R_{2}^{(i)}$ are updated, and the row is locked. This is performed until all rows have been locked. This ends the inner iterations. The outer iterations repeat the above sequence of inner iterations, unlocking all rows at the beginning, until there is no improvement in the overall net-cut. The procedure records the best partitioning up to now obtained, which is returned as the solution. 
If one needs more than two diagonal blocks (i.e., $k>2$ in (11) or (2)), the $\mathrm{KL}$ algorithm can be recursively applied to any resulting submatrix defined by $R_{1}$ or $R_{2}$, thus eventually obtaining a row partition $R_{1}, R_{2}, \ldots, R_{k}$.

Uncoarsening phase. During this phase the partitioning of $A^{(r)}$ is projected to the original matrix through matrices $A^{(r-1)}, A^{(r-2)}, \ldots, A^{(1)}, A^{(0)}$. Two rows $i_{1}$ and $i_{2}$ collapsed in a single one in $A^{h}$ belonging to $R_{p}, 1 \leq p \leq k$, will appear as two different rows of $R_{p}$ in $A^{h-1}$. Optionally, any new matrix $A^{h}$ can be refined with the $\mathrm{KL}$ algorithm.

The KL algorithm itself can be applied to the matrix $A$ (i.e., as if we had a single level algorithm, or equivalently with a coarsening phase with $r=0$ ). However, as it will be shown in Section 3 , it is computationally expensive, because of the large number of rows $m$ of $A$, and can provide unsatisfactory partitionings. On the other hand, applied in combination with a multilevel approach (i.e, coarsening and uncoarsening phases) it is extremely efficient, and the partitioning is significantly improved. Note that the coarsening phase collapses rows with the largest edge-weight, and such rows are expected to be in the same subset $R_{p}$ in a solution, which is exactly what the uncoarsening phase does.

The multilevel algorithm above described has been implemented in $\mathrm{C}$, in a library named UMOA (unconstrained matrix ordering algorithm), which is roughly made of 3100 lines of code. The package can be freely obtained from the authors. It has been optimized using efficient data structures for the coarsening and uncoarsening phases (i.e., AVL trees), as well as for the computation and updating of gains and net-cuts in the KL algorithm. Among the several features of the package, we mention that: (i) when $k>2$ the package allows full control to the user about how to recursively obtain the additional subsets $R_{p}$ from $R_{1}$ and $R_{2}$; (ii) it can be used to obtain both primal and dual block-angular structures (11) and (2). (We note that the reordered matrix in primal block-angular form is not equivalent to the transpose of the reordered matrix in dual block-angular form.)

\section{Reordering Tabular Data Instances}

We applied the multilevel reordering algorithm of Section 2 to a subset of the CSPLIB test suite, a set of instances for tabular data protection (Fischetti and Salazar 2001), plus to additional large instances ("five20b", and "five20c"). CSPLIB can be freely obtained from http://webpages.ull.es/users/casc/\#CSPlib:. CSPLIB contains both low-dimensional artificially generated problems, and real-world highly structured ones. Some of the complex instances were contributed by National Statistical Agencies - as, e.g., Centraal Bureau voor de Statistiek (Netherlands), Energy Information Administration of the Department of Energy (U.S.), Office for National Statistics (United Kingdom) and Statistisches Bumdesant (Germany).

Table 1 shows the features of the instances considered. The small CSPLIB instances were omitted. Column "Name" shows the instance identifier. Columns " $n$ ", " $m$ " and "N. coef" provide, respectively, the number of columns (cells), 
Table 1. Dimensions of the largest CSPLIB instances

\begin{tabular}{lrrr}
\hline Name & $n$ & $m$ & N.coef \\
\hline bts4 & 36570 & 36310 & 136912 \\
five20b & 34552 & 52983 & 208335 \\
five20c & 34501 & 58825 & 231345 \\
hier13 & 2020 & 3313 & 11929 \\
hier13x13x13a & 2197 & 3549 & 11661 \\
hier13x7x7d & 637 & 525 & 2401 \\
hier16 & 3564 & 5484 & 19996 \\
hier16x16x16a & 4096 & 5376 & 21504 \\
jjtabeltest & 3025 & 1650 & 7590 \\
nine12 & 10399 & 11362 & 52624 \\
nine5d & 10733 & 17295 & 58135 \\
ninenew & 6546 & 7340 & 32920 \\
targus & 162 & 63 & 360 \\
toy3dsarah & 2890 & 1649 & 9690 \\
two5in6 & 5681 & 9629 & 34310 \\
\hline
\end{tabular}

rows (additivity constraints) and nonzero coefficients of the constraints matrix $A$ to be reordered.

Tables 2, 3and 4show, respectively, the results obtained reordering the matrices in dual block-angular form for $k=2$, in primal block-angular form for $k=2$, and in dual-block angular form for $k=4$ and 8. Last two columns of Tables 2 and 3 provide information for the single level algorithm (i.e., KL algorithm was applied to the whole matrix). Columns " $m_{1}$ ", " $n_{1}$ ", " $m_{2}$ " and " $n_{2}$ " of tables 2 and 3 provide the number of rows $m_{i}$ and columns $n_{i}$ of the two diagonal blocks.

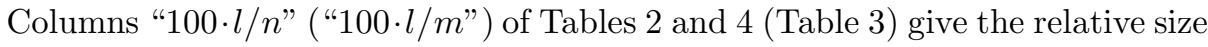
of the linking columns (constraints) block. Columns "CPU" of the three tables report the seconds of $\mathrm{CPU}$ time required to compute the reordering. The runs were carried on a standard PC running Linux with an AMD Athlon 1600+ at $1.4 \mathrm{GHz}$ and $320 \mathrm{MB}$ of RAM. Therefore, the reordered matrix can be efficiently obtained without the need of sophisticated computational resources.

From Tables 24 it can be concluded that:

- The multilevel approach is instrumental in reordering tabular data constraints. It is not only one order of magnitude faster that the single level approach, but also provides much better reorderings (i.e., the linking block becomes much narrower). In particular, all the matrices could be reordered in few seconds on a desktop computer.

- The sizes of the diagonal blocks are similar, which may be a benefit for an optimization solver. This is also instrumental if parallel computations want to be exploited.

- The size of the linking block is instance dependent (from $7.4 \%$ in instance "bts4" of Table2 to 59.8\% in instance "hier16" of Table3). Thus, in principle, not all the reordered matrices are appropriate for a specialized solver for structured problems. 
Table 2. Results for dual block-angular ordering with $k=2$

\begin{tabular}{|c|c|c|c|c|c|c|c|c|}
\hline \multirow[b]{3}{*}{ Name } & \multicolumn{6}{|c|}{ Multilevel } & \multicolumn{2}{|c|}{ Single level } \\
\hline & \multicolumn{2}{|c|}{$A_{1}$} & \multicolumn{2}{|c|}{$A_{2}$} & \multirow{2}{*}{\multicolumn{2}{|c|}{$100 \cdot l / n \mathrm{CPU}$}} & \multirow{2}{*}{\multicolumn{2}{|c|}{$100 \cdot l / n \mathrm{CPU}$}} \\
\hline & $m_{1}$ & $n_{1}$ & $m_{2}$ & $n_{2}$ & & & & \\
\hline bts4 & 17838 & 16898 & 18472 & 16937 & 7.4 & 2 & 21.4 & 53 \\
\hline five20b & 25196 & 14343 & 27787 & 15586 & 13.3 & 6 & 48.4 & 597 \\
\hline five 20 c & 28820 & 14034 & 30005 & 15093 & 15.5 & 7 & 54.3 & 416 \\
\hline hier13 & 1656 & 583 & 1657 & 563 & 43.2 & 0 & 45.0 & 0 \\
\hline hier $13 \times 13 \times 13 a$ & 1890 & 774 & 1659 & 526 & 40.8 & 0 & 46.0 & 0 \\
\hline hier13x13x7d & 792 & 419 & 651 & 218 & 46.1 & 0 & 37.8 & 0 \\
\hline hier16 & 2486 & 740 & 2998 & 1069 & 49.2 & 1 & 55.2 & 1 \\
\hline hier16x16x16a & 2614 & 1074 & 2762 & 1138 & 45.9 & 0 & 45.2 & 0 \\
\hline jjtabeltest & 849 & 1269 & 801 & 1383 & 12.3 & 0 & 15.1 & 0 \\
\hline ninenew & 3007 & 2115 & 4333 & 3085 & 20.5 & 0 & 40.5 & 1 \\
\hline nine $5 d$ & 9007 & 4568 & 8288 & 4303 & 17.3 & 1 & 47.1 & 9 \\
\hline nine12 & 5880 & 4249 & 5482 & 4098 & 19.7 & 3 & 35.5 & 3 \\
\hline targus & 19 & 26 & 44 & 92 & 27.1 & 0 & 21.6 & 0 \\
\hline toy3dsarah & 741 & 1118 & 908 & 1389 & 13.2 & 0 & 24.7 & 0 \\
\hline two5in6 & 5344 & 1749 & 4285 & 1502 & 42.7 & 0 & 56.7 & 3 \\
\hline
\end{tabular}

Table 3. Results for primal block-angular ordering with $k=2$

\begin{tabular}{|c|c|c|c|c|c|c|c|c|}
\hline \multirow{3}{*}{ Name } & \multicolumn{6}{|c|}{ Multilevel } & \multicolumn{2}{|c|}{ Single level } \\
\hline & \multicolumn{2}{|c|}{$A_{1}$} & \multicolumn{2}{|c|}{$A_{2}$} & \multirow{2}{*}{\multicolumn{2}{|c|}{$100 \cdot l / m \mathrm{CPU}$}} & \multirow{2}{*}{\multicolumn{2}{|c|}{$100 \cdot l / m \mathrm{CPU}$}} \\
\hline & $m_{1}$ & $n_{1}$ & $m_{2}$ & $n_{2}$ & & & & \\
\hline bts4 & 16307 & 18389 & 16137 & 18181 & 10.5 & 2 & 21.7 & 37 \\
\hline five20b & 26006 & 17735 & 22576 & 16817 & 12.7 & 4 & 57.9 & 50 \\
\hline five20c & 26693 & 17286 & 26218 & 17215 & 17.1 & 5 & 60.3 & 41 \\
\hline hier13 & 1375 & 1091 & 1076 & 929 & 42.6 & 0 & 53.7 & 0 \\
\hline hier13x13x13a & 1320 & 1075 & 1428 & 1122 & 36.4 & 0 & 51.6 & 0 \\
\hline hier13x13x7d & 483 & 562 & 510 & 621 & 38.0 & 0 & 36.8 & 0 \\
\hline hier16 & 1674 & 1732 & 1676 & 1832 & 59.8 & 0 & 60.4 & 0 \\
\hline hier16x16x16a & 1989 & 2118 & 1676 & 1978 & 41.7 & 0 & 44.6 & 0 \\
\hline jjtabeltest & 737 & 1457 & 686 & 1568 & 7.5 & 0 & 16.8 & 0 \\
\hline ninenew & 3181 & 3239 & 2721 & 3307 & 21.9 & 1 & 46.8 & 1 \\
\hline nine5d & 7111 & 5383 & 7975 & 5350 & 20.5 & 1 & 40.5 & 2 \\
\hline nine12 & 4846 & 5206 & 4666 & 5193 & 17.7 & 1 & 45.6 & 2 \\
\hline targus & 22 & 82 & 22 & 80 & 11.7 & 0 & 19.1 & 0 \\
\hline toy3dsarah & 534 & 1446 & 529 & 1444 & 20.2 & 0 & 20.2 & 0 \\
\hline two5in6 & 3630 & 2816 & 3817 & 2865 & 38.4 & 0 & 37.1 & 0 \\
\hline
\end{tabular}

- In general, the size of the linking block increases with $k$, the number of diagonal blocks. Although the more diagonal blocks, the more "decomposable" becomes the solution of linear systems of equations involving that matrix (see Section 4), the overall solution procedure can become very inefficient due to a large linking block. This tradeoff is usually optimized by small 
Table 4. Results for dual block-angular ordering with $k=4$ and $k=8$

\begin{tabular}{lcrrrr}
\hline & \multicolumn{2}{c}{$k=4$} & & \multicolumn{2}{c}{$k=8$} \\
\cline { 2 - 3 } \cline { 5 - 6 } Name & $100 \cdot l / n \mathrm{CPU}$ & & $100 \cdot l / n \mathrm{CPU}$ \\
\hline bts4 & 15.4 & 3 & & 22.9 & 3 \\
five20b & 34.0 & 7 & & 43.8 & 9 \\
five20c & 26.8 & 9 & & 44.9 & 11 \\
hier13 & 66.9 & 0 & 82.8 & 0 \\
hier13x13x13a & 64.1 & 0 & 80.8 & 0 \\
hier13x13x7d & 71.7 & 0 & 88.6 & 0 \\
hier16 & 73.2 & 1 & & 85.4 & 0 \\
hier16x16x16a & 69.4 & 0 & 83.7 & 0 \\
jjtabeltest & 24.6 & 0 & 36.9 & 0 \\
ninenew & 34.1 & 0 & 54.3 & 0 \\
nine5d & 44.3 & 1 & & 70.6 & 1 \\
nine12 & 31.0 & 1 & & 47.6 & 1 \\
targus & 53.7 & 0 & 54.3 & 0 \\
toy3dsarah & 45.6 & 0 & 68.9 & 0 \\
two5in6 & 73.9 & 0 & 87.2 & 0 \\
\hline
\end{tabular}

values of $k$ (e.g., $k=2,3$ or 4 ), unless the information about the data allows specific larger ones.

- Primal and dual block-angular structures provide linking blocks of different size. The best (primal or dual) ordering is instance dependent, and it seems not to be a clear trend.

The above conclusions are consistent with those obtained in [16 for other types of matrices (for the dual block-angular structure, the only one considered in [16]).

Figures 25 of Appendix A] show the original matrix, 2-blocks dual, 2-blocks primal, and 4-blocks dual reorderings for the four largest instances tested.

\section{Using the Reordered Matrices}

The numerical kernel of any optimization algorithm is to deal with linear systems of equations derived from the constraints matrix. If the block bordered structure of the constraints matrix is exploited, significant savings can be obtained. This is valid for both simplex and interior-point methods, which have been extensively used for cell-suppression, controlled tabular adjustment, and controlled rounding. We will focus on the use of primal block-angular matrices in interior-point methods (which have shown to be the most efficient option for tabular data), and will test them for the controlled tabular adjustment problem.

The main computational burden of an interior-point method [21] is to solve systems of equation with matrix $A \Theta A^{T}$, where $\Theta$ is a diagonal positive definite matrix. For our purposes, and without loss of generality, we will assume $\Theta=I$, thus, the system to be solved is 


$$
\left(A A^{T}\right) \Delta y=g .
$$

This system is named the "normal equations" and it is usually solved by a sparse Cholesky factorization. If $A$ has the structure of (1), we can recast the matrix of system (3) as

$$
A A^{T}=\left[\begin{array}{ccc|c}
A_{1} A_{1}^{T} & & & A_{1} L_{1}^{T} \\
& \ddots & & \vdots \\
& & A_{k} A_{k}^{T} & A_{k} L_{k}^{T} \\
& & \\
\hline & & & \\
L_{1} A_{1}^{T} & \ldots & L_{k} A_{k}^{T} & \sum_{i=1}^{k} L_{i} L_{i}^{T}
\end{array}\right]=\left[\begin{array}{cc}
B & C \\
C^{T} & D
\end{array}\right],
$$

$B, C$ and $D$ being the blocks of $A A^{T}$. Appropriately partitioning $g$ and $\Delta y$ in (3), the normal equations can be written as

$$
\left[\begin{array}{cc}
B & C \\
C^{T} & D
\end{array}\right]\left[\begin{array}{l}
\Delta y_{1} \\
\Delta y_{2}
\end{array}\right]=\left[\begin{array}{l}
g_{1} \\
g_{2}
\end{array}\right]
$$

By eliminating $\Delta y_{1}$ from the first group of equations of (5), we obtain

$$
\begin{aligned}
\left(D-C^{T} B^{-1} C\right) \Delta y_{2} & =\left(g_{2}-C^{T} B^{-1} g_{1}\right) \\
B \Delta y_{1} & =\left(g_{1}-C \Delta y_{2}\right) .
\end{aligned}
$$

Therefore we have reduced the solution of system (3) to the solution of systems with matrix $B$ (which is made of $k$ smallest subsystems $A_{i} A_{i}^{T}, i=1, \ldots, k$ ) and with one system with matrix $\left(D-C^{T} B^{-1} C\right)$, which is named the "Schur complement" of (3). In general, if the solution of systems with $A_{i} A_{i}^{T}, i=1, \ldots, k$ are not too expensive, and the sparsity (the number of nonzero elements) of the Schur complement is not degraded (i.e, decreased too much), we can obtain significant computational savings by solving (6)-(7) instead of (3). Moreover, there are efficient procedures for (6) based on iterative linear solvers 8 .

The above procedure, using a preconditioned conjugate gradient (i.e., iterative solver) for (6) with a specialized preconditioner (see [5]8 for details), has been implemented in an interior-point package for primal block-angular problems [8]. Such procedure can be used for the efficient solution of controlled tabular adjustment (CTA) problems, once the tabular data constraints have been previously reordered as shown in Section 3 Table 5 reports preliminary computational results with an early implementation of CTA based on the specialized interior-point algorithm of [8]. For each of the instances of Table 1-but the two largest ones, that failed with the iterative solver-we solved systems (6) (7) from some interior-point iterations with a sparse Cholesky factorization (the standard procedure used by general interior-point solvers, such as CPLEX) and with the specialized procedure of 8 . Column "Ratio time" of Table 5 show the ratio between both solution times, i.e., how many times faster is the specialized procedure compared to the standard one. We note that the problems were not 
Table 5. Ratio time for the solution of CTA by an interior-point method without and with exploitation of structure

\begin{tabular}{lr}
\hline Name & Ratio time \\
\hline bts4 & 1.5 \\
hier13 & 12.7 \\
hier13x13x13a & 11.6 \\
hier13x13x7d & 3.9 \\
hier16 & 43.5 \\
hier16x16x16a & 43.2 \\
jjtabeltest & 0.7 \\
ninenew & 7.5 \\
nine5d & 2.8 \\
nine12 & 5.1 \\
targus & 1.0 \\
toy3dsarah & 6.0 \\
two5in6 & 10.9 \\
\hline
\end{tabular}

solved up to optimality with the approach of [8], since that procedure has still to be tuned for problems like CTA (which has, for instance, equality linking constraints instead of the inequality ones considered in [8]). However those figures are a good indicator of the expected overall performance in the solution of CTA by exploiting the constraints structure in an interior-point method.

\section{Conclusions}

The structure detection tool used in this work for constraints of tabular data can provide significant computational savings for CTA, and, in general, for any tabular data protection method that has to solve a sequence of linear programming subproblems. Many additional tasks have still to be done. Among them, the main one is to tune the specialized approach of 8 for fully exploiting the reordered matrix, and for obtaining an optimal solution to CTA in a fraction of the time needed by a general solver.

\section{References}

1. Benders, J.F.: Partitioning procedures for solving mixed-variables programming problems. Computational Management Science 2 (2005) 3-19

2. Bixby, R.E.: Solving real-world linear programs: a decade and more of progress. Operations Research 50 (2002) 3-15

3. Bradley, S.P, Hax, A.C., Magnanti, T.L.: Applied Mathematical Programming. Addison-Wesley, Reading (1977).

4. Castro, J.: Network flows heuristics for complementary cell suppression: an empirical evaluation and extensions. Lect. Notes in Comp. Sci. 2316 (2002) 59-73. Volume Inference Control in Statistical Databases, ed. J. Domingo-Ferrer, Springer, Berlin 
5. Castro, J.: Quadratic interior-point methods in statistical disclosure control. Computational Management Science 2 (2005) 107-121

6. Castro, J.: Minimum-distance controlled perturbation methods for large-scale tabular data protection. European Journal of Operational Research 171 (2006) 39-52

7. Castro, J.: A shortest paths heuristic for statistical disclosure control in positive tables. To appear in INFORMS Journal on Computing. Available as Research Report DR 2004/10 Dept. of Statistics and Operations Research, Universitat Politècnica de Catalunya, 2004

8. Castro, J.: An interior-point approach for primal block-angular problems. To appear in Computational Optimization and Applications (2007). Available as Research Report DR 2005/20 Dept. of Statistics and Operations Research, Universitat Politècnica de Catalunya, 2005

9. Cox, L.H.: Network models for complementary cell suppression. J. Am. Stat. Assoc. 90 (1995) 1453-1462

10. Cox, L. H., George, J. A.: Controlled rounding for tables with subtotals. Annals of Operations Research 20 (1989) 141-157

11. Dandekar, R.A.: personal communication (2005)

12. Dandekar, R.A., Cox, L.H.: Synthetic tabular Data: an alternative to complementary cell suppression, manuscript, Energy Information Administration, U.S.

13. Dantzig, G.B., Wolfe, P.: Decomposition principle for linear programs. Operations Research 8 (1960) 101-111

14. Fischetti, M., Salazar, J.J.: Solving the cell suppression problem on tabular data with linear constraints. Management Science 47 (2001) 1008-1026

15. Gondzio, J., Sarkissian, R.: Parallel interior point solver for structured linear programs. Mathematical Programming 96 (2003) 561-584

16. Hu, Y.F., Maguire, K.C.F., Blake, R.J.: A multilevel unsymmetric matrix ordering algorithm for parallel process simulation. Computers and Chemical Engineering 23 (2000) 1631-1647

17. Hundepool, A.: The CASC project. Lect. Notes in Comp. Sci. 2316 (2002) 172 180. Volume Inference Control in Statistical Databases, ed. J. Domingo-Ferrer, Springer, Berlin

18. Karypis, G., Kumar, V.: A fast and high quality multilevel scheme for partitioning irregular graphs. SIAM Journal on scientific Computing 20 (1999) 359-392.

19. Kernighan, B.W., Lin, S.: An efficient heuristic procedure for partitioning graphs. Bell Systems Technical Journal 49 (1970) 291-308

20. Salazar, J.J.: Controlled rounding and cell perturbation: statistical disclosure limitation methods for tabular data. Mathematical Programming 105 (2006) 583-603

21. Wright, S.J.: Primal-Dual Interior-Point Methods. SIAM, Philadelphia (1997). 


\section{A Sparsity Pattern of Original and Reordered Matrices}

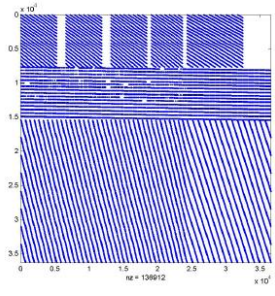

a)

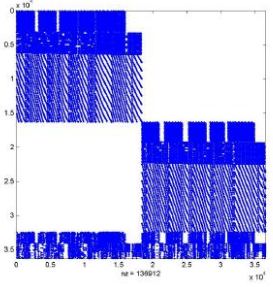

c)

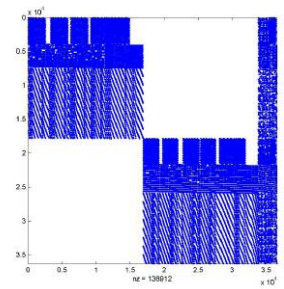

b)

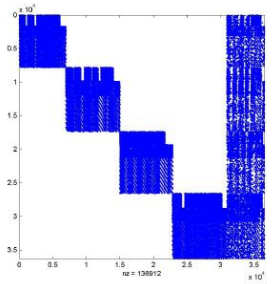

d)

Fig. 2. Results for instance bts4: a) original matrix; b) reordered matrix in dual 2blocks-angular form; c) reordered matrix in primal 2-blocks-angular form; d) reordered matrix in dual 4-blocks-angular form

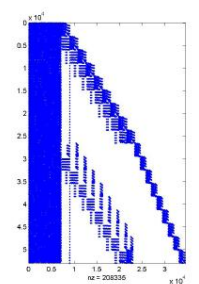

a)

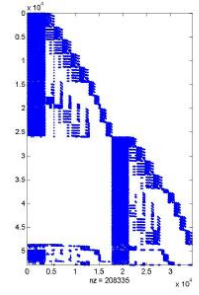

c)

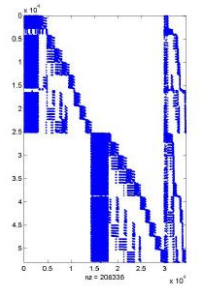

b)

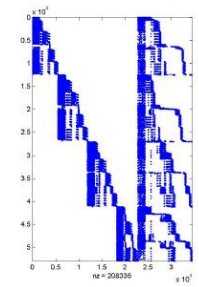

d)

Fig. 3. Results for instance five20b: a) original matrix; b) reordered matrix in dual 2blocks-angular form; c) reordered matrix in primal 2-blocks-angular form; d) reordered matrix in dual 4-blocks-angular form 


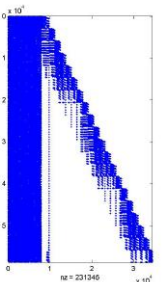

a)

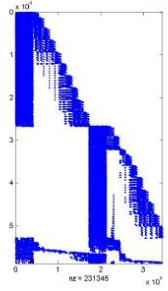

c)

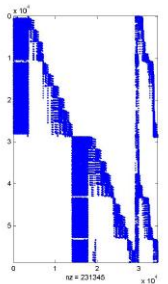

b)

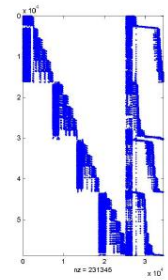

d)

Fig. 4. Results for instance five20c: a) original matrix; b) reordered matrix in dual 2blocks-angular form; c) reordered matrix in primal 2-blocks-angular form; d) reordered matrix in dual 4-blocks-angular form

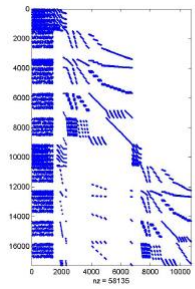

a)

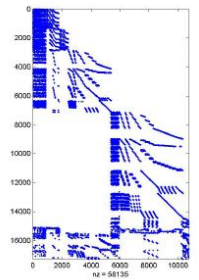

c)

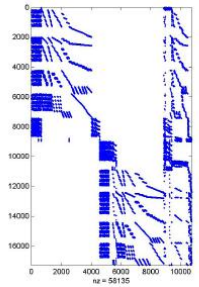

b)

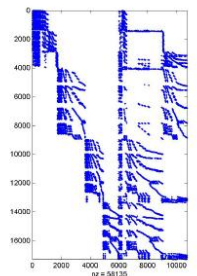

d)

Fig. 5. Results for instance nine5d: a) original matrix; b) reordered matrix in dual 2blocks-angular form; c) reordered matrix in primal 2-blocks-angular form; d) reordered matrix in dual 4-blocks-angular form 